\title{
Carp cDNA sequence encoding a putative diazepam-binding inhibitor/endozepine/acyl-CoA-binding protein ${ }^{1}$
}

\author{
Jean-Leon Chang, Huai-Jen Tsai * \\ Institute of Fisheries Science, National Taiwan Unicersity, Taipei 106, Taiwan \\ Received 19 June 1996; revised 21 August 1996; accepted 10 September 1996
}

\begin{abstract}
A full-length cDNA coding for common carp diazepam-binding inhibitor (DBI)/endozepine (EP)/acyl-CoA-binding protein (ACBP) was isolated and sequenced. The deduced DBI/EP/ACBP is comprised of 87 amino acids (including initiating methionine) without possessing a signal peptide. Common carp DBI/EP/ACBP displays 77\%, 78\%, 70\%, 63\%, $61 \%$ and $45 \%$ identity with human, bovine, rat, frog, duck and yeast DBI/EP/ACBP, respectively.

Keywords: Acyl-CoA-binding protein; Benzodiazepine receptor; cDNA cloning; Diazepam-binding inhibitor; Endozepine; (Carp); (C. carpio)
\end{abstract}

Benzodiazepines are widely used as antianxiety agents, antiepileptic drugs and muscle relaxants in humans. The effects of central type benzodiazepine agonists are mediated by the gamma-aminobutyric acid (GABA)-ergic neurotransmission system, in which GABA acts by increasing chloride permeability in postsynaptic membranes [1]. An endogenous ligand for the benzodiazepine receptor was isolated from human and bovine brain [2], pig upper intestine [3] and rat liver [4]. This ligand is an 86-amino-acid polypeptide designated diazepam-binding inhibitor (DBI)/endozepine (EP)/acyl-CoA-binding protein (ACBP), and is $93 \%$ conserved between humans and bovines [5]. The exact biological function of

\footnotetext{
Corresponding author, Fax: +88623638483

' The nucleotide sequence data reported in this paper have been submitted to the ENBL Data Libraries under accession number L77976.
}

$\mathrm{DBI} / \mathrm{EP} / \mathrm{ACBP}$ is unclear because it has numerous potential endocrine, neuroendocrine and intracrine functions. However, it has been suggested that the physiological roles of DBI/EP/ACBP are fundamental to organisms ranging from yeast to humans based on the highly conserved nature of $\mathrm{DBI} / \mathrm{EP} / \mathrm{ACBPs}$ in evolutionally diverse organisms [6]. To further understand the comparative physiology and evolution of these benzodiazepine receptor ligands at the molecular level, cDNA sequences have been determined for rat [7], human and bovine [8], duck [9], frog [10] and yeast [11] DBI/EP/ACBPs. However, neither the amino acid nor the nucleotide sequences of DBI/EP/ACBP have ever been determined in the lowest vertebrate even though immunological studies revealed the presence of $\mathrm{DBI} / \mathrm{EP} / \mathrm{ACBP}$ in the brain and pituitary of rainbow trout [12]. Here, we report the characterization of cDNA encoding DBI/EP/ACBP of the common carp (Cyprinus carpio), a common fish in aquacul- 
ture worldwide. This is the first sequence of $\mathrm{DBI} / \mathrm{EP} / \mathrm{ACBP}$ in a fish.

A cDNA library was constructed in bacteriophage lambda gt10 using tissue from the carp eye cup, including the retina and optic nerve. A putative recombinant phage clone coding for fish $\mathrm{DBI} / \mathrm{EP} / \mathrm{ACBP}$ was isolated using an oligodeoxynucleotide probe. The probe was a nucleotide sequence correspondent to a highly conserved amino-acid region of human and bovine DBI/EP/ACBPs, which was amino acids located at 51 to 60 . We designed a degenerate primer of 32 different oligonucleotides, which was $5^{\prime}$ GG(C/T)AA(A/G)GG(C/T)AA(A/G)TGGGA(C/ $\mathrm{T})-3^{\prime}$. The inserted DNA fragment was extracted from a recombinant phage by $E c o \mathrm{Rl}$ digestion. The DNA fragment was then subcloned into EcoRI-digested pBluescript. In addition to T7 and T3 primers, two primers were synthesized for determining the sequences of the inserted DNA by following the dideoxynucleotide-chain-termination method [13] with a Sequenase kit (US Biochemical, Cleveland, $\mathrm{OH})$. Primers OP1 ( $+45,5^{\prime}$-CAAC GGACGCTGAGATGCT $-3^{\prime},+63$, sense strand) and OP2 $(+282$, 5'-CTCT TCCACCTTAGCGAT-3', + 265, antisense strand) were used.

Fig. 1 shows the nucleotide and the deduced amino-acid sequences of the cloned DBI/EP/ACBP

\section{AAGCACTTCGTTATACAGTAACAGACTGAAACATCTACAACC}

42

ATG TCT GAG GCT GAG TTT CAG AAA GCA GCA GAG GAG GTC AAA 84 met ser glu ala glu phe gln lys ala ala glu glu val lys 14

CAG CTG AAA GCG AAA CCA ACG GAC GCT GAG ATG CTG GAA ATT 126 gln leu lys ala lys phe thr asp ala glu met leu glu ile 28

TAC AGT CTG TAC AAA CAG GCC ACC GTA GGA GAT GTT AAC ACA 168 tyr ser leu tyr lys gln ala thr val glu asp val asn thr $\mathbf{4 2}$

GCT CGA CCG GGC ATG TTA GAT TTC ACT GGC AAG GCC AAA TGG 210 ala arg phe gly met leu asp phe thr gly lys ala lys trp 56

GAT GCT TGG GAG GCC AAG AAA GGT ATG AGT AAG GAT GAT GCT 252 glu ala trp glu ala lys lys gly met ser lys asp asp ala 70

ATG AAA GCA TAC ATC GCT AAG GTG GAA GAG CTG AAG GGG AAA 294 met lys ala tyr ile ala lys val glu glu leu lys gly lys 84

TAC GGA ATC TAA TAGACGGACCTATCATGGCCAGCCTGTCTTTACCTGTA 345 tyr gly ile *

CTGTTCTCCAATAAAGTGCCTTCAGGCCTCAAGTGTGGCCCCTCACAAGGGTGTT 400 ACAGTTATGCTGAAGCATGCAGACTCCGTTCCTGAAGGAGAGATAGGCACATTC 455 CTTGCAGGCCAAAAGAAATTGTCCACCACTAACCACATGTAGTGAAAGCCCTAAT 510

Fig. 1. Nucleotide sequence and deduced amino-acid sequence of carp DBI/EP/ACBP cDNA. The nucleotides were numbered beginning with the first nucleotide at the $5^{\prime}$ end. The number on the second line at the end of each row, indicates the cumulative number of amino acids. The polynucleotide signal is underlined and the stop codon is marked with an asterisk.

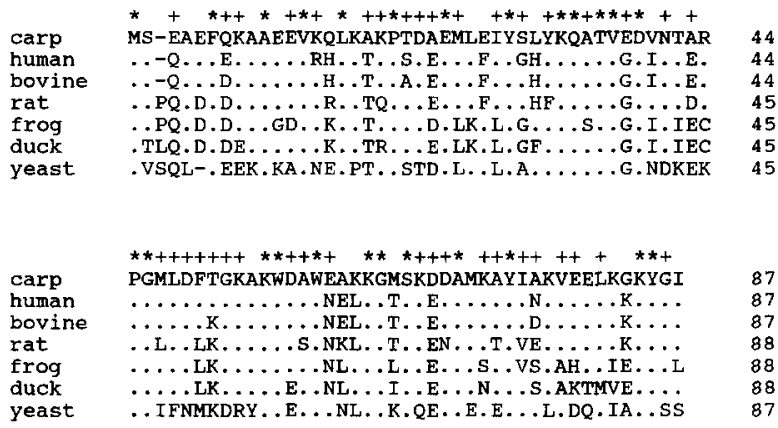

Fig. 2. Comparison of the predicted amino-acid sequences of carp DBI /EP / ACBP with those of human and bovine [8], rat [7], frog [10], duck [9] and yeast [11]. Numbering begins at the initial mRNA codon. Identical amino-acid residues are indicated by dots $(\cdot)$; an asterisk $\left({ }^{*}\right)$ indicates that an amino acid is perfectly conserved among all known species; and a plus sign $(+)$ indicates that an amino acid is well conserved.

cDNA. This 510 nucleotide long cDNA had a $5^{\prime}$ untranslated sequence of 42 nucleotides, a single open reading frame of 261 nucleotides and a $3^{\prime}$ short untranslated region of 207 nucleotides, followed by a poly(A) tail. The polyadenylation signal, AATAAA, was located 150 nucleotides upstream of the start of the $\operatorname{poly}(\mathrm{A})$ tail.

The deduced carp DBI/EP/ACBP is an 87 amino-acid polypetide (including the initial methionine). The amino-acid sequences of human, bovine, rat, frog, duck and yeast DBI/EP/ACBP display $77 \%, 78 \%, 70 \%, 63 \%, 61 \%$ and $45 \%$ identity with carp DBI/EP/ACBP, respectively (Fig. 2). Like all other DBI/EP/ACBPs sequenced thus far, the amino-acid sequence of carp DBI/EP/ACBP is not derived from a precursor molecule containing a transient signal peptide. Several aromatic residues were highly conserved, such as Phe-6, Tyr-29, Trp-56, Trp-59, Tyr-74 and Tyr-85. The lysine residues flanking octadecaneuropeptide and trikontatetraneuropeptide (Lys-33, Lys-53 and Lys-55) were also highly conserved during evolution.

We found that carp DBI/EP/ACBP, like those of human, bovine and yeast, lacked the dibasic sequences found in frog and duck DBI/EP/ACBPs [10]. But in carp DBI/EP / ACBP Lys-62 and Lys-63 were conserved, which was the right flank side of dibasic sequences. Unlike frog and duck DBI/EP/ACBPs, which have additional Pro-2 and Cys-44 residues, no Pro and Cys were found in carp 
$\mathrm{DBI} / \mathrm{EP} / \mathrm{ACBP}$ at the corresponding positions. Furthermore, carp DBI/EP/ACBP displayed some unique characteristics. Three polar (acidic or basic) amino-acid residues present in all other known DBI/EP/ACBPs were replaced by non-polar residues in carp DBI/EP/ACBP. Glu-23, Glu-42 and Lys-83 in mammal, frog and duck DBI/EP/ACBPs were replaced by Ala-23, Ala-42 and Gly-83 in carp DBI/EP/ACBP. In contrast, a non-polar residue, Gly-38, found in all other known DBI/EP/ACBPs, was replaced by a polar residue, Glu-38, in carp. The biological significance of these amino-acid alternations deserve further study.

This study was supported by grants from National Science Council, Republic of China, under NSC-852611-B-002-003.

\section{References}

[1] Costa, E. and Guidotti, A. (1979) Annu. Rev, Pharmacol. Toxicol. 19, 531-545.
[2] Shoyab, M., Gentry, L.E., Marquardt, H. and Todaro, G.J. (1986) J. Biol. Chem. 261, 11968-11973.

[3] Chen, Z., Agerberth, B., Gell, K., Anderson, M., Mutt, V., Ostenson, C., Efendic, S., Barrossoderling, J., Persson, B. and Jornvall, H. (1988) Eur. J. Biochem. 174, 239-245.

[4] Knudsen, J., Hojrup, P., Hansen, H.O., Hansen, H.F., and Roepstorff, P. (1989) Biochem. J. 262, 513-519.

[5] Marquardt, H., Todaro, G.J. and Shoyab, M. (1986) J. Biol. Chem. 26, 9727-9731.

[6] Tonon, M.C., Adjeroud, S., Lamacz, M., Louiset, E., Danger, J.M., Desrues, L., Cazin, L., Nicolas, P. and Vaudry, H. (1989) Neuroscience 31, 485-493.

[7] Mocchetti, I., Einstein, R. and Brosius, J. (1986) Proc. Natl. Acad. Sci. USA 83, 7221-7225.

[8] Webb, N.R., Rose, T.M., Malik, N., Marquardt, H., Shoyab, M., Todaro, G.J. and Lee, D.C. (1987) DNA 6, 71-79.

[9] Rose, T.M., Schultz, E.R., Sasaki, G.C., Kolattukudy, P.E. and Shoyab, M. (1994) DNA Cell Biol. 13, 669-678.

[10] Lihrmann, I., Plaquevent, J.-C., Tostivint, H., Raijmakers, R., Tonon, M.-C., Conlon, J.M. and Vaudry, H. (1994) Proc. Natl. Acad. Sci. USA 91, 6899-6903.

[11] Rose, T.M., Shultz, E.R. and Todaro, G.J. (1992) Proc. Natl. Acad. Sci. USA 89, 11287-11291.

[12] Malagon, M., Vallarino, M., Tonon, M.C. and Vaudry, H. (1992) Brain Res. 576, 208-214.

[13] Sanger, F., Nicklen, S. and Coulsen, A.R. (1977) Proc. Natl. Acad. Sci. USA 74, 5463-5467. 